\title{
AVALIAÇÃO DA QUALIDADE DE VIDA E DO SONO DE ATLETAS PARALÍMPICOS BRASILEIROS
}

\author{
EVALUATION OF THE QUALITY OF LIFE AND SLEEP IN BRAZILIAN PARALYMPIC ATHLETES
}

EVALUACIÓN DE LA CALIDAD DE VIDA Y SUEÑO DE ATLETAS PARALÍMPICOS BRASILEÑOS

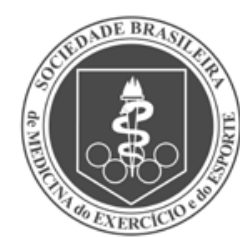

Artigo Original
Andrea Maculano Esteves ${ }^{1}$ (Educadora Física)

Andressa Silva ${ }^{2,5}$ (Fisioterapeuta)

Amaury Barreto 3 (Biólogo)

Daniel Alves Cavagnolli ${ }^{3}$

(Educador Físico)

Luciana Santo Andrea Ortega ${ }^{4}$

(Educadora Física)

Andrew Parsons ${ }^{5}$ (Jornalista)

Edilson Rocha Tubiba ${ }^{5}$

(Educador Físico)

Murilo Barreto ${ }^{5}$ (Educador Físico)

Ciro Winckler de Oliveira Filho

(Educador Físico)

Sergio Tufik (Médico)

Marco Túlio de Mello 2,5

(Educador Físico)

1. Faculdade de Ciências Aplicadas, Universidade Estadual de Campinas, Limeira, SP, Brasil

2. Universidade Federal de Minas Gerais, Departamento de Esportes, Belo Horizonte, MG, Brasil

3. Universidade Federal de

São Paulo, Departamento de Psicobiologia, São Paulo, SP, Brasil 4. Associação Fundo de Incentivo à Pesquisa (AFIP), São Paulo, SP, Brasil 5. Comitê Paralímpico Brasileiro (CPB), Brasília, DF, Brasil

\section{Correspondência:}

Marco Túlio de Mello.

Departamento de Esportes - Escola de Educação Física, Fisioterapia e Terapia Ocupacional - UFMG.

Avenida Presidente Carlos Luz, 4664, Campus Pampulha - Belo Horizonte, Minas Gerais, Brasil, CEP: 31310-250, tmello@demello.net.br

\section{RESUMO}

Introdução: o esporte paralímpico brasileiro vem ganhando destaque no cenário mundial e, com isso, a avaliação de variáveis que possam influenciar positivamente no desempenho desses atletas é de suma importância para o acompanhamento durante o seu período de treinamento. Objetivo: avaliar a qualidade de vida e do sono de atletas paralímpicos brasileiros. Métodos: foram estudados 49 atletas paralímpicos das modalidades natação $(n=20)$ e atletismo $(n=29)$. Os atletas responderam a questionários que avaliaram seu padrão e queixas de sono e qualidade de vida. Resultados: a maioria dos atletas $(65,30 \%)$ relatou má qualidade do sono, visto que a latência do sono neste grupo foi significativamente maior do que em atletas com boa qualidade de sono. Cinquenta por cento dos atletas relataram o desejo de fazer mudanças em seu horário de sono e 52\% gostariam de aumentar o seu tempo de sono. A sonolência diurna excessiva foi observada em $53,06 \%$ dos atletas. Quanto às queixas de distúrbios do sono, foram relatados chute ou espasmos das pernas e ronco. Menores índices de qualidade de vida foram encontrados no meio ambiente em comparação com os domínios físicos, psicológicos ou sociais. Conclusões: os resultados sugerem que a maioria dos atletas apresentou uma má qualidade do sono e, consequentemente, um alto índice de insatisfação com o sono. Além disso, menores escores de qualidade de vida foram encontrados no domínio ambiental, que está relacionado com a segurança física, proteção e condições em casa.

Palavras-chave: esporte, sono, qualidade de vida, atividade física, distúrbios do sono.

\section{ABSTRACT}

Introducction: Brazilian paralympic sports are gaining prominence on the world stage. That's why the evaluation of some characteristics that may influence positively the athletes' performance is so important to be observed during the training sessions. Objective: to assess the quality of life and sleep quality of Brazilian paralympic athletes. Methods: 49 paralympic athletes participating in swimming $(n=20)$ and athletics $(n=29)$ were studied. The athletes answered questionnaires assessing their sleep patterns and complaints and their quality of life. Results: most athletes (65,30\%) reported poor sleep quality, and the sleep latency in this group was significantly greater than in the athletes with good sleep quality. Fifty percent of the athletes reported a desire to make changes in their sleep schedule and 52\% would like to increase their sleep time. Excessive daytime sleepiness was observed in 53.06\% of the athletes. Regarding complaints of sleep disorders, there were reported kicking or jerking of the legs and snore. Lower scores for quality of life were found in the environment if compared to the physical, psychological or social relationship domains. Conclusion: most athletes reported poor sleep quality and, consequently, a high index of dissatisfaction with their sleep. Moreover, lower quality of life scores werefound in the environmental domain, which is related to physical safety, protection and conditions at home.

Keywords: sports, sleep, quality of life, physical activity, sleep disorders.

\section{RESUMEN}

Introducción: El deporte paralímpico brasileño viene ganando destaque en el escenario mundial y con eso la evaluación de variables que puedan influenciar positivamente en el desempeño de esos atletas es de suma importancia para el acompañamiento durante su período de entrenamiento. Objetivo: Evaluar la calidad de vida y del sueño de atletas paralímpicos brasileños. Métodos: Fueron estudiados 49 atletas paralímpicos de las modalidades natación $(n=20)$ y atletismo $(n=29)$. Los atletas respondieron a cuestionarios que evaluaron su estándar y quejas de sueño y calidad de vida. Resultados: La mayoría de los atletas (65,30\%) relató mala calidad del sueño, visto que la latencia del sueño en este grupo fue significativamente mayor que en atletas con buena calidad de sueño. Cincuenta por ciento de los atletas relató el deseo de hacer cambios en su horario de sueño, y a 52\% le gustaría aumentar su tiempo de sueño. La somnolencia diurna excesiva fue observada en $53,06 \%$ de los atletas. Cuanto a las quejas de disturbios del sueño, fueron relatadas patadas o espasmos de las piernas y ronquido. Menores valores de calidad de vida fueron encontrados en el medio ambiente en comparación con los dominios físicos, psicológicos o sociales. Conclusiones: Los resultados sugieren que la mayoría de los atletas presentó una mala calidad del sueño y, consiguientemente, un alto índice de insatisfacción con el sueño. Además, menores valores de calidad de vida fueron encontrados en el dominio ambiental, que está relacionado con la seguridad fisica, protección y condiciones en casa.

Palabras clave: deporte, sueño, calidad de vida, actividad física, disturbios del sueño. 


\section{INTRODUÇÃO}

O esporte paralímpico brasileiro vem apresentando um crescente avanço no cenário mundial. Diante desse panorama, a necessidade de avaliações nessa população específica também vem se tornando imprescindível para acompanhar a evolução desse esporte.

A relação entre o sono e o exercício físico pode sofrer influência de fatores como nível de aptidão física inicial, intensidade, duração do tempo e o tipo de exercício físico realizado ${ }^{1,2}$. Estudos demonstram que as contrações musculares exigidas na execução de qualquer exercício físico são estímulos capazes de promover depleção dos estoques de energia e provocar micro-lesões, ou seja, uma alta atividade catabólica durante a vigília que exige uma alta atividade anabólica durante o sono, em especial, durante o sono de ondas lentas (SOL) onde ocorre a maior liberação de hormônio do crescimento (GH) em especial em um terço da noite de sono ${ }^{3,4}$.

No entanto, o tempo gasto dos atletas com a prática das modalidades esportivas, bem como, com a prática do exercício físico também podem interferir em outros fatores da vida, como, a qualidade de vida $(\mathrm{QV})^{5}$.

A qualidade de vida é definida pela Organização Mundial da Saúde (OMS) como: "[...] a percepção do indivíduo de sua posição na vida no contexto da cultura e sistema de valores nos quais ele vive e em relação aos seus objetivos, expectativas, padrões e preocupações". Esta definição deixa implícita a ideia do conceito subjetivo, multidimensional e inclui elementos de avaliação tanto positivos como negativos. Também reflete a subjetividade do construto inserida no contexto cultural, social e do meio ambiente ${ }^{6}$.

Estudos têm mostrado que o exercício físico pode influenciar positivamente na qualidade de vida tanto em indivíduos com alguma patologia ${ }^{7,8}$ quanto em populações saudáveis ${ }^{9}$. No entanto, grandes volumes de treinamento físico podem acarretar em alterações negativas, principalmente devido à relação entre o desgaste físico e psicológico que ocorre com o treinamento/competições comparado ao tempo que o atleta tem ou consegue descansar. Dessa forma, o volume de treinamento pode apresentar uma relação dose-resposta negativa entre nível de atividade física e qualidade de vida ${ }^{10,11}$. Em adição, já é demonstrado na literatura que a qualidade do sono está intrinsecamente ligada à QV, onde alterações no seu padrão normal podem levar a prejuízos físicos e psicológicos na saúde ${ }^{12}$.

Neste contexto, a necessidade de avaliar o sono e a qualidade de vida de atletas paralímpicos é importante, uma vez que exercem grande influência no desempenho dessa população. O objetivo do presente estudo foi avaliar o perfil da qualidade de vida e do sono de atletas paralímpicos brasileiros.

\section{MATERIAIS E MÉTODOS}

Foram avaliados 49 atletas paralímpicos brasileiros de ambos os gêneros, portadores de deficiência visual e física, participantes das modalidades natação $(n=20)$ e atletismo $(n=29)$. O atletas se encontravam no perido de preparação para os Jogos Parapanamericanos de Guadalara 2011. A pesquisa foi aprovada pelo Comitê de Ética da Universidade Federal de São Paulo, SP, Brasil, (CEP \#0294/11) e todos os atletas assinaram o Termo de Consentimento Livre e Esclarecido concordando em participar voluntariamente da pesquisa.

Os atletas foram entrevistados e avaliados nas dependências do Centro de Estudos em Psicobiologia e Exercício (CEPE) da Associação Fundo e Incentivo a Pesquisa (AFIP), São Paulo, SP, Brasil. Os atletas foram encaminhados a uma sala reservada e silenciosa para responderam aos questionários relacionados à avaliação do perfil do sono (Pittisburg, Questionário do Sono UNIFESP e Epworth) e da qualidade de vida (WHOQOL BRIEF).
Questionário utilizado como instrumento para a avaliação subjetiva da qualidade do sono ${ }^{13}$. Composto por 19 itens pontuados em uma escala de 0 a 3 . Os itens são alocados em sete grupos, sendo: (1) qualidade subjetiva do sono; (2) latência do sono; (3) duração do sono; (4) eficiência habitual do sono; (5) alterações do sono; (6) uso de medicações do sono; (7) disfunção diurna. A partir da somatória dos escores dos sete grupos é determinado o IQSP. O resultado do IQSP varia entre 0 e 21, sendo classificado respectivamente como qualidade de sono boa (0 a 4), qualidade de sono ruim (5 a 10) e indicação de distúrbio do sono (acima de 10).

Questionário do Sono da UNIFESP: No Questionário do Sono da UNIFESP ${ }^{14}$ foram analisadas as questões sobre características e queixas de hábitos relacionados ao sono, como o tempo de ir para a cama e levantar-se, a natureza dos problemas de sono, e a frequência de sua ocorrência.

Escala de Sonolência de Epworth: Escala mais utilizada atualmente para a avaliação subjetiva da sonolência diurna, sendo capaz de diferenciar as pessoas com e sem sonolência das com sonolência excessiva. O Epworth consiste em oito questões que descrevem situações diárias que podem induzir à sonolência. Cada questão é graduada de zero a três pontos sendo que escores acima de 10 inferem de sonolência diurna significativa e acima de 15 estão associados à sonolência patológica presente em condições específicas, tais como apnéia do sono e narcolepsia ${ }^{15}$.

Questionário de Qualidade de Vida (WHOQOL Bref): O WHOQOL-bref (World Health Organization Quality of Life Instrument) consta de 26 questões, sendo duas questões gerais de qualidade de vida e as demais 24 representam cada uma das 24 facetas que compõe o instrumento original (domínios físico, psicológico, relações sociais e meio ambiente). Para cada questão existem 5 graus de intensidade e o paciente opta por uma delas ${ }^{16}$.

\section{Análise estatística}

Os dados foram analisados pelo programa Statistics for Windows 7.0 (StaSoft, INC ${ }^{\oplus}$ ). Inicialmente foi realizado um teste de normalidade dos dados utilizando o teste Komolgorov-Smirnov. Foi utilizado o teste de Mann-Whitney para amostras independentes não paramétricas e a ANOVA para medidas repetidas para os domínios da qualidade de vida. O nível de significância considerado para todos os testes foi de $p<0,05$ e os resultados estão apresentados em média \pm desvio padrão.

\section{RESULTADOS}

Os resultados referentes as características antopométricas dos atletas (dividida por gênero) estão descritas na tabela 1.

Os resultados demonstraram que a maioria dos atletas apresentou uma pobre qualidade de sono (sono ruim) avaliada pelo questionário de Pittisburg (65,30\%), com um aumento significativo na latência do sono quando comparados aos atletas que tiveram uma boa qualidade do sono (sono bom) $(Z=2,02 p<0,04)$ (figura 1 ).

Foi relatada sonolência excessiva diurna em $53,06 \%$ dos atletas. Em relação às características do sono 50\% relataram querer mudar alguma coisa no seu horário de dormir, visto que estes apresentaram maiores escores no Questionário de Pittsburgh $(6,25)$ quando comparados aos que não gostaria de alterar seu horário de dormir $(4,09)(Z=3,60$, $p<0,001)$. Dentre as mudanças no horário de dormir, $52 \%$ gostariam de aumentar o seu tempo de sono. No que se refere às queixas de distúrbios do sono, 12,24\% relataram chutar ou movimentar bruscamente as pernas durante o período de sono e 18,36\% relataram roncar pelo menos entre uma a duas vezes por mês.

Não foram encontradas diferenças significantes nas variáveis relacionadas ao sono entre as modalidades avaliadas (natação e atletismo).

A qualidade do sono não influenciou nos resultados na qualidade de vida, ou seja, os atletas que relataram qualidade de sono ruim 
não apresentaram piores escores nos domínios da qualidade de vida. No entanto, foi verificada uma redução nos escores do domínio meio ambiente em relação aos domínios físico, psicológico e relações sociais respectivamente, avaliados pelo questionário WHOQOL Bref (Anova $\mathrm{p}<0.05$ ) (figura 2).

Foi demonstrado também uma redução nos escores do domínio meio ambiente nas mulheres $(13,41)$ em relação aos homens $(15,21)$ na modalidade natação $(Z=2,10, p<0,04)$.

Tabela 1. Características antopométricas dos atletas.

\begin{tabular}{c|c|c}
\hline Característica & Feminino & Masculino \\
\hline Idade $($ anos $)$ & $27,27 \pm 8,33$ & $25,92 \pm 7,06$ \\
\hline Peso $(\mathrm{kg})$ & $55,53 \pm 12,04$ & $68,76 \pm 13,56$ \\
\hline Altura $(\mathrm{cm})$ & $157,73 \pm 16,62$ & $174,61 \pm 10,37$ \\
\hline
\end{tabular}

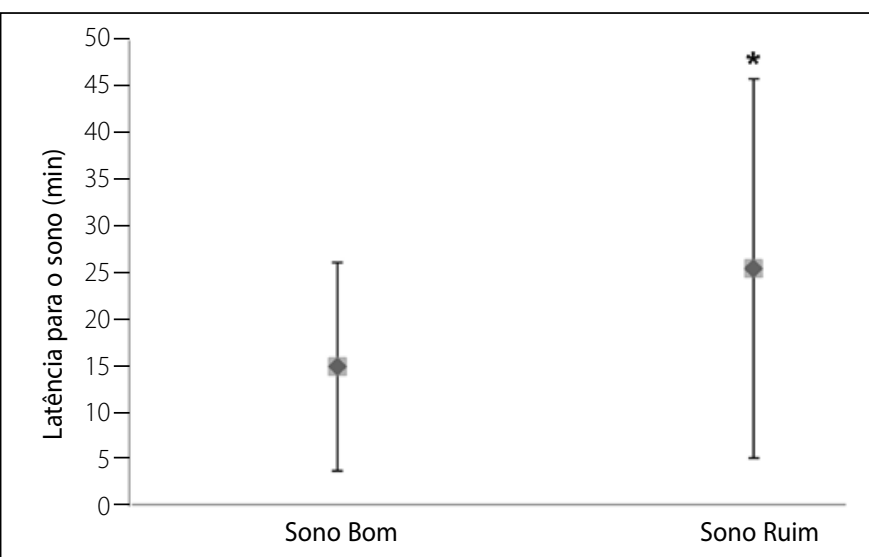

Figura 1. Comparação da latência do sono entre os atletas que apresentaram uma boa qualidade de sono (sono bom) e uma pobre qualidade de sono (sono ruim) avaliada pelo Questionário de Pittsburgh. Mann-Whitney, $\mathrm{p}<0,05$.

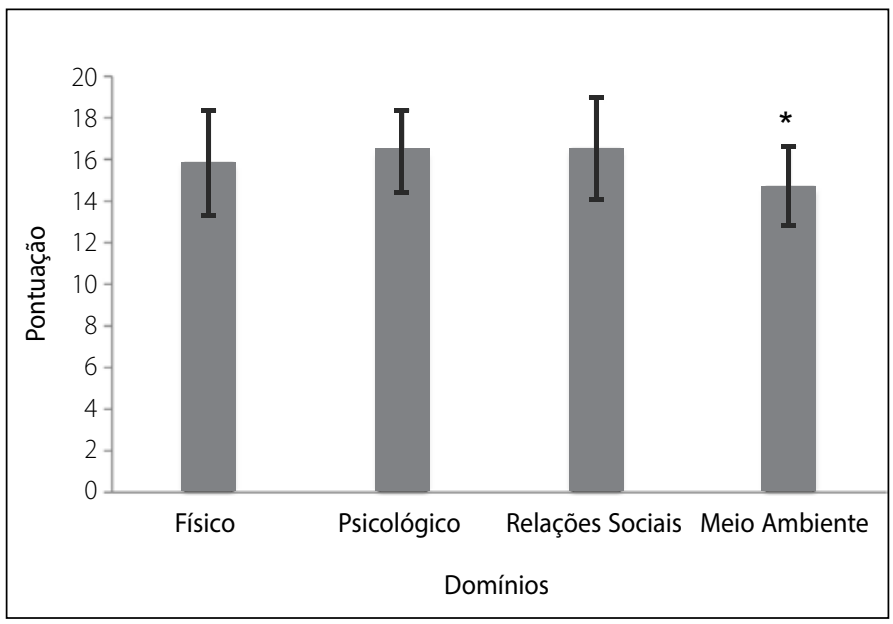

Figura 2. Comparação dos domínios da qualidade de vida avaliados pelo Questionário de WHOQOL Bref. Anova, $p<0.05$. ${ }^{*}$ difere dos domínios físico, psicológico e relações sociais.

\section{DISCUSSÃO}

Um dos principais achados do presente estudo foi que a maioria dos atletas paralímpicos analisados de atletismo e natação apresentou uma pobre qualidade do sono avaliado pelo questionário de Pittsburgh, tendo como consequência a presença de sonolência excessiva diurna em mais da metade dos atletas.

Talvez esses achados sejam um reflexo da redução no processo de recuperação, onde o sono pobre e de baixa qualidade e eficiência levariam a uma redução da capacidade física do atleta. Silva et al. ${ }^{17}$ demonstraram em estudo que a maioria dos atletas (73,5\%) da delegação brasileira paralímpica durante o mundial paralímpico de atletismo de 2011 apresentou queixas musculoesqueléticas. Hobson ${ }^{18}$ descreveu que existe uma relação em forma de " $U$ " invertido entre a fadiga induzida pelo exercício e a qualidade do sono, onde exercícios em altas intensidades e/ou em excesso, assim como um sono de má qualidade, com períodos insuficientes de recuperação ou distúrbios do sono, acarretam em prejuízo no rendimento do atleta.

Silva et al. ${ }^{19}$ demonstraram que 83\% dos 27 atletas paralímpicos analisados antes dos Jogos Paraolímpicos de Pequim apresentaram má qualidade do sono antes das competições. Essas informações fortalecem a hipótese de que o tipo de treinamento físico realizado pode influenciar no padrão de sono do atleta e que informações como a programação de treinamento, descanso e os tempos de competição devem ser sempre levados em consideração durante a fase preparatória do atleta.

Já estão bem consolidadas na literatura as alterações que o exercício físico provoca no padrão de sono. Entre elas podemos destacar o aumento do sono de ondas lentas ${ }^{20-23}$, aumento da latência para o sono $\mathrm{REM}^{22,24}$ e a redução da latência para o início do sono quando não há alteração recuperação pode não haver alterações nestas variáveis ${ }^{2,23,25,26}$. Os resultados do presente estudo demonstraram que os atletas com uma pobre qualidade do sono apresentaram um aumento na latência do sono, sugerindo mais uma vez que fatores extrínsecos (como por exemplo, relação entre intensidade do treinamento e períodos de recuperação) ou intrínsecos (como por exemplo, distúrbios do sono) talvez estejam influenciando nessa má qualidade do sono. Foi demonstrado também que 30\% dos atletas apresentaram queixas relacionadas ao sono, como movimentação brusca das pernas durante o sono e ronco. De Mello et al. ${ }^{27-29}$ avaliaram por meio da polissonografia que lesados medulares apresentavam movimentos periódico de pernas (MPP) e que o exercício físico agudo (teste de esforço máximo) e o exercício crônico (treinamento por 44 dias no limiar ventilatório 1) reduziram significativamente o MPP durante o sono. No entanto, essa presença da queixa de movimentos durante o sono nos atletas do presente estudo talvez esteja relacionado ao desequilíbrio entre volume/intensidade do treinamento e a recuperação dos atletas, visto que nos estudos de De Mello et al. ${ }^{27-29}$ o exercício físico imposto obedecia às regras de periodização, ou seja, períodos de sobrecarga e descanso regulares.

Em relação ao perfil da qualidade de vida dos atletas, foi verificado que o domínio meio ambiente (relacionado a fatores como segurança física e proteção) ${ }^{16}$ apresentou menores escores em relação aos domínios físico, psicológico e relações sociais. Como a qualidade de vida engloba vários aspectos (psicológicos, biológicos e sociais), é de suma importância a percepção e compreensão do indivíduo como um todo sobre esses aspectos. O atleta paraolímpico brasileiro, na maioria das vezes, apresenta um nível sócio econômico baixo, e esse contexto sócio-cultural, no qual o atleta está inserido, influencia diretamente na sua forma de pensar, sentir e agir. Assim, essa redução no domínio meio ambiente na qualidade de vida nos sugere a necessidade de intervenções de órgãos responsáveis para a obtenção de uma melhora nesta questão, uma vez que, este domínio refere-se a fatores que não podem ser controlados individualmente.

\section{CONCLUSÃO}

Os resultados do presente estudo demonstraram que a maioria dos atletas (65,30\%) apresentou uma pobre qualidade de sono, bem como, um alto índice de insatisfação com o seu próprio sono. Além disso, a redução no domínio meio ambiente da qualidade de vida relata preocupações em relação às questões de segurança física, proteção e ambiente do lar. 


\section{AGRADECIMENTOS}

A Associação de Fundo e Incentivo à Pesquisa (AFIP), a Fundação de Amparo à Pesquisa do Estado de São Paulo (FAPESP - CEPID n ${ }^{\circ}$ 98/14303-3 to ST), ao Centro de Estudos em Psicobiologia e Exercício (CEPE), ao Centro de Estudos Multidisciplinar em Sonolência e Aci- dentes (CEMSA), ao Comitê Paralímpico Brasileiro (CPB) e a Academia Paralímpica Brasileira (APB).

Todos os autores declararam não haver qualquer potencial conflito de interesses referente a este artigo.

\section{REFERÊNCIAS}

1. Viana VA, Esteves AM, Boscolo RA, Grassmann V, Santana MG, Tufik S, et al. The effects of a session of resistance training on sleep patterns in the elderly. Eur J Appl Physiol. 2012;112(7):2403-8.

2. Flausino NH, Da Silva Prado JM, de Queiroz SS, Tufik S, de Mello MT. Physical exercise performed before bedtime improves the sleep pattern of healthy young good sleepers. Psychophysiology. 2012;49(2):186-92.

3. Driver HS, Taylor SR. Exercise and sleep. Sleep Med Rev. 2000;4(4):387-402.

4. Dattilo M, Antunes HK, Medeiros A, Mônico Neto M, Souza HS, Tufik S, et al. Sleep and muscle recovery: endocrinological and molecular basis for a new and promising hypothesis. Med Hypotheses. 2011;77(2):220-2

5. Giacobbi PR Jr, Stancil M, Hardin B, Bryant L. Physical activity and quality of life experienced by highly active individuals with physical disabilities. Adapt Phys Activ Q. 2008;25(3):189-207.

6. The World Health Organization Quality of Life assessment (WHOQOL): position paper from the World Health Organization. Soc Sci Med. 1995;41(10):1403-9.

7. Austin S, Qu H, Shewchuk RM. Association between adherence to physical activity guidelines and health-related quality of life among individuals with physician-diagnosed arthritis. Qual Life Res. 2012;21(8):1347-57

8. Aidar FJ, de Oliveira RJ, Silva AJ, de Matos DG, Carneiro AL, Garrido N, et al. The influence of the level of physical activity and human development in the quality of life in survivors of stroke. Health Qual Life Outcomes. 2011:9:89.

9. Bize R, Plotnikoff RC. The relationship between a short measure of health status and physical activity in a workplace population. Psychol Health Med. 2009;14(1):53-61.

10. Brown DW, Balluz LS, Heath GW, Moriarty DG, Ford ES, Giles WH, et al. Associations between recommended levels of physical activity and health-related quality of life. Findings from the 2001 Behavioral Risk Factor Surveillance System (BRFSS) survey. Prev Med. 2003;37(5):520-8.

11. Brown DW, Brown DR, Heath GW, Balluz L, Giles WH, Ford ES, et al. Associations between physical activity dose and health-related quality of life. Med Sci Sports Exerc. 2004;36(5):890-6.

12. Lopes C, Esteves AM, Bittencourt LR, Tufik S, Mello MT. Relationship between the quality of life and the severity of obstructive sleep apnea syndrome. Braz J Med Biol Res. 2008;41(10):908-13.

13. Buysse DJ, Reynolds CF 3rd, MonkTH, Berman SR, Kupfer DJ. The Pittsburgh Sleep Quality Index: a new instrument for psychiatric practice and research. Psychiatry Res. 1989;28(2):193-213.

14. Pires ML, Benedito-Silva AA, Mello MT, Pompeia Sdel G, Tufik S. Sleep habits and complaints of adults in the city of São Paulo, Brazil, in 1987 and 1995. Braz J Med Biol Res. 2007;40(11):1505-15.

15. Smith SS, Oei TP, Douglas JA, Brown I, Jorgensen G, Andrews J. Confirmatory factor analysis of the Epworth Sleepiness Scale (ESS) in patients with obstructive sleep apnoea. Sleep Med. 2008 Oct;9(7):739-44
16. Fleck MP, Louzada S, Xavier M, Chachamovich E, Vieira G, Santos L, et al. Application of the Portuguese version of the abbreviated instrument of quality life WHOQOL-bref. Rev Saude Publica. 2000;34(2):178-83.

17. Silva A, Matiello SM, Peterson R, Zanca GG, Vital R, Winckler C, et al. Queixas musculoesqueléticas e procedimentos fisioterapêuticos na delegação brasileira paralímpica durante o mundial paralímpico de atletismo em 2011. Rev Bras Med Esporte. 2013;19(4):256-9.

18. Hobson JA. Sleep after exercise. Science. 1968;162(3861):1503-5.

19. Silva A, Queiroz SS, Winckler C, Vital R, Sousa RA, Fagundes V, et al. Sleep quality evaluation, chronotype, sleepiness and anxiety of Paralympic Brazilian athletes: Beijing 2008 Paralympic Games. Br J Sports Med. 2012;46(2):150-4.

20. Youngstedt SD, O'Connor PJ, Crabbe JB, Dishman RK. The influence of acute exercise on sleep following high caffeine intake. Physiol Behav. 2000;68(4):563-70.

21. Shapiro CM, Bortz R, Mitchell D, Bartel P, Jooste P. Slow-wave sleep: a recovery period after exercise Science. 1981;214(4526):1253-4

22. Horne JA, Moore VJ. Sleep EEG effects of exercise with and without additional body cooling. Electroencephalogr Clin Neurophysiol. 1985;60(1):33-8.

23. Hague JF, Gilbert SS, Burgess HJ, Ferguson SA, Dawson D. A sedentary day: effects on subsequent sleep and body temperatures in trained athletes. Physiol Behav. 2003;78(2):261-7.

24. Youngstedt SD, O'Connor PJ, Dishman RK. The effects of acute exercise on sleep: a quantitative synthesis. Sleep. 1997;20(3):203-14

25. Driver HS, Rogers GG, Mitchell D, Borrow SJ, Allen M, Luus HG, et al. Prolonged endurance exercise and sleep disruption. Med Sci Sports Exerc. 1994;26(7):903-7.

26. Matsumoto J, Nihisho T, Suto T, Sadahiro T, Miyoshi M. Influence of fatigue on sleep. Nature. 1968;218(5137):177-8

27. De Mello MT, Esteves AM, Tufik S. Comparison between dopaminergic agents and physical exer cise as treatment for periodic limb movements in patients with spinal cord injury. Spinal Cord. 2004:42(4):218-21

28. De Mello MT, Lauro FA, Silva AC, Tufik S. Incidence of periodic leg movements and of the restless legs syndrome during sleep following acute physical activity in spinal cord injury subjects. Spinal Cord. 1996;34(5):294-6.

29. De Mello MT, Silva AC, Esteves AM, Tufik S. Reduction of periodic leg movement in individuals with paraplegia following aerobic physical exercise. Spinal Cord. 2002;40(12):646-9. 